\title{
Gruppenmedien und Verkündigung: Ein Diskussionsbeitrag
}

\author{
von Manuel Olivera
}

Im November 1977 wird in München der erste Internationale Kongreß über „Gruppenmedien und Evangelisation" stattfinden. Er steht unter der Schirmherrschaft der Päpstlichen Kommission für die soziale Kommunikation und wird von $\mathrm{OCIC}^{1}$ in $\mathrm{Zu}$ sammenarbeit mit Unda ${ }^{2}$ organisiert. Das Thema, der Zeitpunkt, die internationalen Zusammenkünfte und die daran beteiligten Institutionen bieten eine einzigartige Möglichkeit, eine Reihe von koordinierten Studien in die Wege zu leiten, um ein klares Bild von den Möglichkeiten und Grenzen zu erhalten, die sich den Gruppenmedien auf dem Gebiet der Vertiefung der Glaubenserziehung bieten. In diesem Sinn ist das Wort "Evangelisation" hier zu verstehen".

In dieser kurzen Arbeit will ich mich darauf beschränken, die Gruppenkommunikationsmedien und deren Hauptmerkmale in groben Zügen aufzuzeigen, so, wie ich sie aufgrund meiner bisherigen Erfahrungen sehe. Zum Schluß möchte ich noch erläutern, welche Vorstellungen ich von einer ersten Untersuchung auf diesem Gebiet habe.

\section{Die Gruppenmedien}

Bereits seit mehreren Jahren verfolgen wir bei den Medien der sozialen Kommunikation die Entwicklung einer neuen Form, die in Lateinamerika, zumindest seit dem Seminar von Santa Inés (Peru, 1966), mit dem Namen „Gruppenmedien“ im Gegensatz zu Massenmedien bezeichnet wird. Darunter versteht man im Rahmen eines Filmforums gezeigte Filme, durch Schallplatten oder Kassetten verbreitete Hörspiele, die zu anschließenden Diskussionen in kleinen Gruppen anregen, Artikel in Zeitungen oder Zeitschriften, die in Arbeitsgruppen analysiert werden, Diapositive und Bilder, die zu Instruktion und Meditation dienen, Theateraufführungen, in denen Probleme angeschnitten und die Zuschauer zur Mitarbeit und Lösung aufgerufen werden, Lieder, in denen eine Lebensauffassung zum Ausdruck kommt und die zu Diskussionen und Gedankenaustausch anregen usw.

Diese besondere Form hat rasche Verbreitung gefunden und ist unter verschiedenen Bezeichnungen, wie z. B. "medios grupales" auf spanisch, „animation media" auf englisch, "moyens légers" auf französisch, "producciones para foros" (Produktionen für Diskussionsforen) ${ }^{4}$ usw. bekannt, oder aber man bezeichnet sie nach der Art der Verwendung oder Gestaltung, wie z. B. Film-, Theater- oder Schallplattenforen, Hörbilder, Hörspiele, Psychodramen usw.

In einigen Dokumenten wird diese Form bereits erwähnt und für die pastorale Arbeit empfohlen. Das bedeutendste ist eine Studie über „Evangelisation und Gruppenmedien", die DECOS-CELAM im Jahre 1972 herausgebracht hat ${ }^{5}$. Darin heißt es, daß diese Gruppenmedien „den Dialog von Mensch zu Mensch begünstigen... das Publikum zur aktiven Mitarbeit und zum Nachdenken anregen ... und die Botschaft

P. Manuel Olivera S. J. ist Vizepräsident der Katholischen Weltvereinigung für Rundfunk, Unda, und Direktor von Unda-Lateinamerika. 
dem einzelnen nicht in einer individuellen Form, sondern als Glied einer Gruppe oder Gemeinschaft übermitteln..." „Wenngleich sie auch kein breites Publikum erreichen, so gewährleisten sie aber eine tiefgreifende Formung, sind ein geeignetes Mittel zur Veranschaulichung und Erklärung der christlichen Lehre mit all ihren Konsequenzen und ermöglichen eine freie und bewußte Entscheidung für eine persönliche Bindung an Christus. " "Es ist dringend notwendig, sie in die Katechese miteinzubeziehen, ... denn sie sind für die religiöse Erziehung hervorragend geeignet."

Das Auftauchen dieser Gruppenmedien ist kein isoliertes Phänomen. Es geht Hand in Hand mit der theologischen Erneuerung, die das II. Vatikanische Konzil, und in Lateinamerika Medellín, einleiteten, mit einer Pastoral, die sich auf die Gemeinden als Grundpfeiler stützt, mit der neuen, von Pablo Freire angeregten Sicht der Erziehung und mit den Bestrebungen zahlreicher christlicher Kommunikatoren, die ihre eigene Arbeit durch die kommerzielle Struktur der großen Massenmedien beschränkt sehen und nach verbesserten Möglichkeiten suchen.

Werden wir nun, nach mehrjähriger Erfahrung, in der Lage sein, die Hauptcharakteristika dieser Gruppenmedien so herauszuarbeiten, daß sie gleichzeitig eine anschauliche Definition bilden?

\section{Charakteristika}

Vier Hauptmerkmale sind für das System der Gruppenmedien von entscheidender Bedeutung. Wenn auch nicht alle immer in idealer Weise zusammenwirken, so zeigen sie doch die Grundlinien des Systems auf.

1. Sie wenden sich an Gruppen. Im allgemeinen handelt es sich dabei um Gruppen mit begrenzter Teilnehmerzahl, so daß jeder einzelne seinen persönlichen Beitrag zu dem Thema leisten kann, das gemeinschaftlich besprochen wird. Die Gruppenmedien suchen weder eine heterogene, pluralistische Menge noch das einzelne, in sich abgeschiedene Individuum anzusprechen.

2. Sie regen zum Dialog an. Ziel der Gruppenmedien ist es, den Gedanken- und Erfahrungsaustausch innerhalb der Gruppe zu fördern. Daher auch die im Englischen gebräuchliche Bezeichnung "animation media“. Die Hauptbestrebung liegt in der aktiven Mitarbeit der Teilnehmer. Sie sollen von bloßen passiven Zuschauern in aktive Gestalter ihres eigenen Lebens verwandelt werden.

3. Sie bemühen sich, ein Thema tiefgründig zu behandeln. Einerseits haben die Autoren alle Möglichkeit, soweit die Produktionsart dies zuläßt, die Problematik darzustellen. Andererseits aber werden auch die Zuhörer, bzw. Zuschauer angeregt, das Thema in einem anschließenden Gedankenaustausch noch weiter auszuschöpfen und zu vertiefen.

4. Sie fördern die persönlichkeitsbildenden Neigungen des einzelnen. Die vermassenden Neigungen des Menschen haben die Tendenz, Gedanken, Werte, Geschmack und Handlungsweisen einer Uniformierung und Standardisierung zu unterstellen und Entscheidungen auf andere abzuwälzen. Durch die persönlichkeitsbildenden Neigungen wird der Mensch angehalten, sein Leben, sowohl als Individuum als auch als Teil einer Gemeinschaft, bewußt und verantwortungsvoll zu gestalten. Da die Gruppenmedien die persönlichkeitsbildenden Neigungen fördern, werden sie im DECOSDokument auch als „Nicht-Massemedien“ bezeichnet. 
$\mathrm{Zu}$ diesen vier Grundmerkmalen kann man noch weitere Eigenschaften hinzufügen, die sich ableiten lassen, wenn man die Produktionen betrachtet, mit denen die Gruppenmedien arbeiten.

5. Die Werke haben meist einen anspruchsvollen Inhalt. Es handelt sich bei ihnen weder ausschließlich um leichte Unterhaltung noch um Programme rein informativer, künstlerischer, humoristischer oder folkloristischer Art. Sie bringen ein für die Diskussionsrunde wichtiges Thema, ein Problem, das die Gruppe vital berührt. Meist sind auch Kommentare, Anweisungen oder Leitfäden beigefügt, die helfen sollen, das Thema in Reflexionsgruppen zu vertiefen.

6. Sie pflegen manches „offenzulassen“, um so die Diskussion noch besser zu entfachen, das heißt, es sind keine voll "abgerundeten" Werke, in denen bereits alles ausgesprochen, erklärt und erläutert ist und die nur noch vom Zuhörer aufgenommen und im Gedächtnis gespeichert zu werden brauchen. Es handelt sich auch nicht um schulmeisterliche Ausführungen über ein schwieriges Thema, mit denen sich der Verfasser brüstet, sondern um einen Beitrag, der innerhalb der Gruppe erwogen, diskutiert und analysiert werden soll.

7. Sie sind leicht zu verbreiten. Sie sind sowohl vom finanziellen als auch vom technischen Gesichtspunkt aus für die Gruppen (in Schulen, Gemeinden, Familien, etc.) erschwinglich. An Vorführungsapparaten gibt es eine Vielzahl verschiedener Modelle, deren Verbreitung davon abhängt, wie preisgünstig und populär jeweils die neuen Geräte sind. (Daher die Bezeichnung "moyens légers", die in Kanada gebräuchlich ist.)

8. Sie sind im allgemeinen kurz, das heißt, sie lassen Zeit für eine anschließende Diskussion. Diese ist in ihrer Länge, entsprechend der Zeit, die den Teilnehmern zur Verfügung steht, flexibel.

9. Viele Werke entstammen Produktionen, die ursprünglich für die großen Massenmedien verfaßt wurden. In jüngster Zeit werden allerdings immer mehr Werke eigens für Darbietungen in kleinen Gruppen produziert.

Wenn man schließlich die Gruppenarbeit noch eingehender beachtet, lassen sich vier weitere Charakteristika feststellen:

10. Sie bieten den Basisgruppen die Möglichkeit, sich mit Grundproblemen, vor allem des Gemeinschaftslebens, auseinanderzusetzen und Lösungen ausfindig zu machen. In den Werken werden Themen aufgeworfen, bestimmte Fragen hervorgehoben, das Verantwortungsbewußtsein gefördert, der Blickwinkel erweitert.

11. Aus diesem Grund ist die Arbeitsmethodik ebenso wichtig wie das Werk selbst. Dabei kommt den Prinzipien der Gruppendynamik, angewandt in diesem Fall auf eine Art asystematischer und gemeinschaftlicher Selbstbildung, besondere Bedeutung zu.

12. Eine wichtige Vermittlerrolle nimmt der Moderator oder Gruppendiskussionsleiter ein. Seine Aufgabe besteht darin, einerseits die Gruppen entsprechend zu leiten, andererseits den größtmöglichen Gewinn aus dem präsentierten Stück herauszuarbeiten.

13. Diese Art von Produktionen wendet sich im allgemeinen an einen Personenkreis mit ausgesprochen pädagogischen Interessen und Fähigkeiten, an Menschen, die sich der Gruppenarbeit in Schulen, Gemeinden und Pfarreien, verschiedenen Organisationen, Gewerkschaften etc. widmen. 


\section{Untersuchung}

Wegen der besonderen Bedeutung der Gruppenmedien sollten die folgenden Untersuchungen unverzüglich durchgeführt werden, um bei Durchführung internationaler Treffen noch eingebracht $z u$ werden:

1. Bestandsaufnahme bezüglich der Gruppenmedien

Vom Organisationskomitee des internationalen Treffens wird bereits ein Verzeichnis von Personen, Institutionen und technischen Hilfsmitteln angefertigt. Hier ist an erster Stelle eine Zusammenarbeit nötig, damit die Umfrage rechtzeitig und umfassend beantwortet wird. Darüber hinaus aber muß diese Arbeit noch in den einzelnen Ländern durch folgende Einzelheiten ergänzt werden:

- Welche Produktionsformen werden verwendet?

- Welchen Inhalt und welche geistige Ausrichtung haben die Werke?

- Welche didaktischen Methoden erfordert der Gebrauch der Programme in den Gruppen?

- Welche Probleme, Bestrebungen, Tendenzen, Bemühungen, Mängel, Perspektiven etc. zeichnen sich $a b$ ?

- Welche Vertriebsmodelle gibt es?

- Welche Ausbildungsmodelle gibt es bereits?

- Wie kann der Austausch verbessert werden?

\section{Intellektuelle Vertiefung des Systems}

Die Verbindung von Gruppenmedien und Evangelisation wirft eine Reihe von Themen auf, die eine Begriffssbestimmung und -klärung wünschenswert machen. Ich nenne hier nur einige, die bereits Gegenstand der Auseinandersetzung sind:

- Was verstehen wir unter „vermassend“ und „persönlichkeitsbildend“, wenn wir vom Gebrauch der Kommunikationsmedien, seien es die großen oder kleinen, sprechen?

- Welche Interpretation des Evangeliums sollen wir fördern? Sind alle Interpretationen gleichwertig und bieten alle dem Menschen unserer Zeit die gleichen Perspektiven?

- Sind die Gruppenmedien nur eine schwache Reaktion der Unterlegenheit und Resignation angesichts des mißglückten Versuchs, sich die großen Massenmedien für die Ziele der Evangelisation zunutze zu machen?

- Welche Beziehung besteht zwischen Evangelisation und menschlicher Promotion, zwischen Zeitlichem und Ewigem? Wie zeigen sich unterschiedliche Auffassungen von der Kirche in der Produktion und Anwendung der Gruppenmedien?

3. Untersuchung über verschiedene praktische Aspekte

Wie gestaltet man geeignete Kurse für Produzenten, Gruppenleiter und Verteiler?

Welche Probleme bringen Markt und Verteilung mit sich, welche treten bei der Koordination und Komplementierung der einzelnen Bestrebungen und Arbeiten auf? 


\section{Modellerfahrung bezüglich der Vertiefung}

Die bemerkenswertesten Beispiele dieser Form der Gruppenarbeit müssen herausgegriffen, systematisch angewandt und mit größtmöglicher, wissenschaftlicher Sorgfalt ausgewertet werden.

\section{Gleichzeitige Verwendung des Gruppensystems und der Massenmedien}

Einige Radioschulen bedienen sich folgender interessanter Methode: Sie verbinden den Gebrauch der Rundfunksender mit der Gruppenarbeit. Diese Methode müßte erläutert und geprïft werden.

6. Arbeitsgruppen auf nationaler Ebene, die das Thema "Gruppenmedien" bearbeiten a) Ich würde zunächst in einzelnen Ländern, Gebieten oder auch nur einigen bedeutenden Städten die Produzenten dazu anregen:

- ihre Werke vorzuführen und zu erklären,

- ein repräsentatives Beispiel ihrer Arbeit aufzuzeigen,

- die Methodik, die ihre Produktion erfordert, zu erläutern,

- die Möglichkeiten und Grenzen dieses Systems zu analysieren.

Auf diese Weise erhält man bereits eine an der Materie interessierte Arbeitsgruppe. Dabei braucht man sich anfangs keineswegs nur auf das Thema "Evangelisation“ zu beschränken, sofern auch andere Parallelthemen zur Verfügung stehen.

Von diesen Tagungen sollte zweckmäßigerweise ein Protokoll verfaßt werden; die Zusammenfassung dieser Niederschriften könnte das gewünschte Investigationsmaterial ergeben.

b) Die weitere Arbeit mit dieser Gruppe hängt vom Einfallsreichtum des Leiters ab. Man muß aber in der gesamten Arbeit auf einem geplanten Einsatz und einer Koordination aller Kräfte bedacht sein, um sich nicht auf lokaler oder regionaler Ebene zu erschöpfen oder sich in nebensächlichen Einzelheiten zu verlieren. Ein Koordinator auf nationaler und kontinentaler Ebene ist unbedingt erforderlich.

c) Für die geistige Vertiefung fehlen noch einige Grundsatzartikel und weitere schriftliche Beiträge. Das Dokument von DECOS-CELAM über „Evangelisation und Massenmedien" ist dafür vielleicht ein Anfang.

Anmerkungen:

1. Office Catholique International du Cinema, Brüssel.

2. Unda (Welle): Internationale katholische Vereinigung für Rundfunk und Fernsehen, Brüssel.

3. Wilhelm Schätzler (Bonn) macht darauf aufmerksam, daß nach „Evangelii Nuntiandi" (Rom 1975) der Begriff der Evangelisation weiter gefaßt werden muß als nur in Richtung der ,Glaubenserziehung ${ }^{c}$, denn die Kirche „evangelisiert, wenn sie sich darum bemüht, allein durch die göttliche Kraft der Botschaft, die sie verkündet, zugleich das persönliche und kollektive Bewußtsein der Menschen, die Tätigkeit, in der sie sich engagieren, ihr konkretes Leben und jeweiliges Milieu umzuwandeln" (Nr. 18). Dieser Aspekt ist sicher nicht zu übersehen und auch bei den folgenden Ausführungen mitzudenken. Dabei verweist Schätzler mit Recht auf die Bedeutung der ,kirchenbildenden Gruppenmedien' hin, 
weil die Kirche nicht allein als Weltkirche existiert, sondern sich auch in Kleingruppen manifestiert. Dieser Aspekt ist auch deswegen wichtig, weil auf diese Weise der emotionale Vorbehalt abgebaut werden könnte, ${ }_{n}$ der den Massenmedien weitgehend als Verkündigungsinstrument entgegengebracht" wird (Brief an den Verf. v. 10.6.1976).

4. Vgl. Josef Hosse: ,Discoforum' Lateinamerika. In: CS 7:1974, 159-165.

5. Veröffentlicht in: CS 7:1974, 170-182.

\section{S U M M A R Y}

Group media are communications media which lead to the discussion of problems and invite the recipients to participate in their solution. They aim at groups, stimulate dialogue, and try to cover a subject in depth. They help to build up personality, have a demanding content, but do not cover a subject totally. They are easy to spread. The presentations are normally short and so give the possibility of discussing the problems to a considerable extent. Therefore, the methodology for their use, as well as the person who moderates, is important. There should be a kind of inventarisation of this media as soon as possible, and its use should be developed more deeply without neglecting the practical aspects. The relation between group media and mass media has also to be studied, as well as the possibilities for national and international co-operation.

\section{RESUME}

Par l'appelation moyens de groupe, on entend les mass media dont l'utilisation attaque des problèmes et invite les spectateurs à coopérer et à résoudre. Ils s'adressent d̀ des groupes, incitent au dialogue et essayent de traiter un thème en profondeur. Ils encouragent les penchants formateurs de personnalité de chacun, ont la plupart du temps un contenu exigeant, ne traitent pas un thème de façon définitive et sont faciles à diffuser. Leurs présentations sont, en général, courtes et offrent par là, la possibilité de réfléchir sur les problèmes abordés. C'est pourquoi la méthode de travail est aussi importante que le modérateur. Un inventaire de ses moyens serait à faire sans retard, le système serait à approfondir de façon intellectuelle, de même qu'il faudrait prendre note des aspects pratiques. Il faudrait examiner la réaction entre les moyens de groupe et les mass media, de même qu'une possibilité de coopération nationale et internationale.

\section{RESUMEN}

Por medios de grupo se entienden los medios de difusión en cuya utilización se analizan problemas y se pide la colaboración del espectador en la busqueda de soluciones. Están orientados a grupos, suscitan el diálogo y tratan de estudiar un tema a fondo. Estimulan las preferencias del individuo en orden a su formación personal, tienen a menudo contenido muy rico, tratan el tema sin agotarlo y son de fácil difusión. En general se trata de programas breves que ofrecen la posibilidad de una confrontación con problemas seleccionados. Por ello es tan importante el método de trabajo como el moderador. Es inaplazable tomar conciencia sobre estos medios, habría que profundizar intelectualmente el sistema teniendo en cuenta al mismo tiempo los aspectos prácticos. También habría que analizar la relación entre medios de grupo y medios de comunioación social para masas, así como la posibilidad de una cooperación nacional e internacional. 